\section{Highly Pathogenic Avian Influenza A(H5N1) Virus among Poultry, Ghana, 2015}

\section{Ivy Asantewaa Asante, Stephanie Bertram, Joseph Awuni, Abraham Nii Okai Commey, Ben Aniwa, William Kwabena Ampofo, Gülsah Gabriel}

Author affiliations: Heinrich Pette Institute, Leibniz Institute for Experimental Virology, Hamburg, Germany (I.A. Asante, S. Bertram, G. Gabriel); Noguchi Memorial Institute for Medical Research, University of Ghana, Accra, Ghana (I.A. Asante, W.K. Ampofo); Center for Structural and Cellular Biology in Medicine at the University of Lübeck, Lübeck, Germany (S. Bertram, G. Gabriel); Veterinary Services Directorate, Accra (J. Awuni, A.N.O. Commey, B. Aniwa)

DOI: http://dx.doi.org/10.3201/eid2212.160639

To the Editor: Outbreaks of highly pathogenic avian influenza (HPAI) A(H5N1) virus among poultry were first reported in Africa in 2006, with initial reports from Nigeria (1). The virus then spread to several countries (e.g., Egypt, Côte d'Ivoire, Burkina Faso, Niger) in Africa, leading to large economic losses $(1,2)$. In 2007, Ghana reported the first HPAI H5N1 cases among poultry in 3 regions: Greater Accra, Volta, and Brong Ahafo $(3,4)$. The outbreak was contained by measures such as destruction of all birds on affected farms, disinfection of affected farms, and restricted movement of poultry and poultry products. Soon after containment, active influenza surveillance was initiated among birds, domestic poultry, and the human population throughout the country (5). Until 2015, no influenza-positive samples among birds had been detected in Ghana since the 2006-2007 outbreak. In January 2015, Nigeria resumed reporting HPAI H5N1positive samples among poultry (6). One month later, HPAI H5N1-positive samples among chickens were confirmed in Burkina Faso (7). Then, in April 2015, chicken farmers in the Greater Accra region in Ghana reported a large number of deaths among domestic chicken flocks. Tracheal swabs collected from dead chickens and tested at the laboratories of the Veterinary Services Directorate in Accra, Ghana, confirmed the presence of HPAI H5N1 virus infection. By June 2015, the poultry populations in 5 of Ghana's 10 regions were affected, leading to the death or culling of $\approx 100,000$ poultry (7). Affected farms in Ghana (in the Greater Accra, Volta, and Ashanti regions) included medium-scale commercial farms with $\approx 30,000$ chickens (broilers and layers) ranging from day-old chicks to layers $>21$ weeks of age; small-scale commercial bird farms with 200-1,000 chickens; and freerange local poultry of mixed species raised with low levels of biosafety. The death rate for chickens during the period of sample collection (April 13-June 11, 2015) was 17.6\% (6,919 of 39,281 poultry died) (7). No direct links among farms were evident at this time. However, further spread in the Greater Accra region has been attributed to movement of live poultry. Outbreaks have been documented in live bird markets and backyard poultry, leading to a ban on movement of live poultry, feed, and equipment from affected regions. These counter-measures resulted in reduced incidence among poultry. For a lower-middle-income country like Ghana, such outbreaks are a major threat to food security and human health. We describe the outbreak strain found in Ghana during 2015 and its zoonotic potential.

We obtained and analyzed sequences for the major viral genes involved in viral pathogenicity, such as the hemagglutinin (HA), polymerase basic protein 2 (PB2), nucleoprotein, and neuraminidase (NA) genes (online Technical Appendix, http://wwwnc.cdc.gov/EID/article/22/12/160639-Techapp1.pdf). Sequence analysis of HA revealed that the 2015 Ghana outbreak strain possessed a multibasic cleavage site (RERRRKR/GLF), which is common for the HPAI H5N1 virus (8). Substitutions (D94N, S133A, S155N, T156A [H5 numbering]) associated with increased virus binding to human-type $\alpha 2$-6-linked sialic acids were detected. Phylogenetic analysis confirmed that the Ghana outbreak strain belonged to clade 2.3.2.1c (Figure, panel A), as reported by the World Organisation for Animal Health in May 2015 (7). The strain clustered with contemporary viruses from Europe, Asia, the Middle East, and other West Africa countries (i.e., Burkina Faso, Côte d'Ivoire, Niger, and Nigeria). HA sequences of viruses isolated from 3 regions (Greater Accra, Volta, Ashanti) in Ghana were highly homologous, comparable to other Ghana viruses isolated in 2015 deposited in the Global Initiative on Sharing Avian Influenza Data (http://platform.gisaid.org) and GenBank databases (Figure, panel A). In total, 9 aa substitutions were detected in the Ghana strains as compared with the HPAI H5N1 virus that caused the 2015 outbreak in Nigeria (online Technical Appendix Table). Genetic analysis of PB2 showed that the Ghana viruses lacked the known human adaptive signatures E627K or D701N, which enable increased replication and virulence in the human host $(9,10)$. Phylogenetic analysis revealed that PB2 of this virus clustered with H9N2 viruses isolated in Asia during 2007-2013 (Figure, panel B). Nucleoprotein sequences of the Ghana 2015 outbreak strain show $99 \%$ homology with the Nigeria 2015 outbreak strain. NA mutations known to reduce susceptibility to oseltamivir in NA H254Y were not observed.

Our phylogenetic analysis suggests that the Ghana HPAI H5N1 strains belong to clade 2.3.2.1c, as was reported for the 2015 Nigeria outbreak. Because of the high homology $(>90 \%)$ between the Ghana and Nigeria strains, the HPAI H5N1 Ghana outbreak strain likely originated in Nigeria (Figure, panel A). Migratory bird 

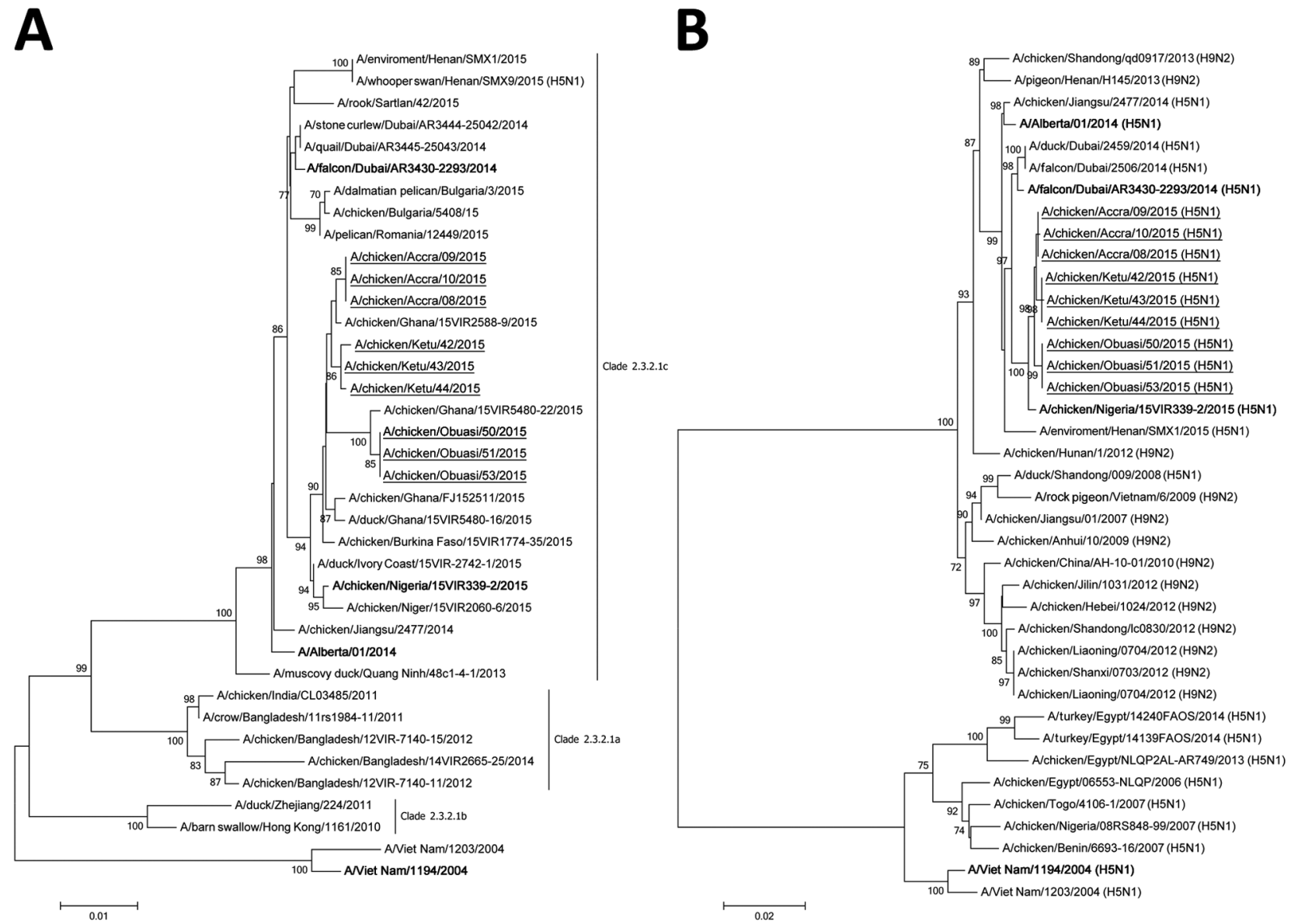

Figure. Phylogenetic analysis of highly pathogenic avian influenza $A(H 5 N 1)$ viruses isolated from poultry in Ghana in 2015: A) hemagglutinin; B) polymerase basic protein 2. Viruses sequenced for this study are underlined and reference viruses are in bold; other sequences were downloaded from the Global Initiative on Sharing Avian Influenza Data (http://platform.gisaid.org) and GenBank databases. Evolutionary analyses were conducted with MEGA6 (http://www.megasoftware.net/). Bootstrap values $>70 \%$ of 500 replicates are shown at the nodes. Scale bars indicate number of nucleotide substitutions per site.

movements and human activities have been implicated in the virus's introduction to the Africa continent. However, intercountry borders in West Africa are known to be porous because of frequent trading activities, possibly accounting for the spread of the virus in the subregion of West Africa.

Because the HPAI H5N1 virus of clade 2.3.2.1c has previously caused deaths in humans, the potential risk for transmission from infected poultry to humans is a major concern. Increased vigilance and rapid implementation of countermeasures are required to mitigate further virus adaptation and potential outbreaks among humans.

\section{Acknowledgments}

We thank the director and head of the laboratory of the Veterinary Services Directorate in Accra, Ghana, for their support. We thank Carola Dreier, Géraldine Engels, and Swantje Thiele at the Heinrich Pette Institute for constructive discussions about methods and sequence analysis. We are also grateful to the director and staff of the Virology Department at the Noguchi Memorial Institute for Medical Research, University of Ghana, for support with sample collection and shipment of samples.

\section{References}

1. Cattoli G, Monne I, Fusaro A, Joannis TM, Lombin LH, Aly MM, et al. Highly pathogenic avian influenza virus subtype H5N1 in Africa: a comprehensive phylogenetic analysis and molecular characterization of isolates. PLoS ONE. 2009;4:e4842. http://dx.doi.org/10.1371/journal.pone.0004842

2. Peiris JSM, de Jong MD, Guan Y. Avian influenza virus (H5N1): a threat to human health. Clin Microbiol Rev. 2007;20:243-67. http://dx.doi.org/10.1128/CMR.00037-06

3. Danso EF, Ampofo W, Afari E, Wurapa F, Aryee M, Koney E, et al. Avian influenza surveillance in domestic poultry and wild bird-Tema Metropolis, Ghana, 2010. Journal of Commonwealth Veterinary Association. 2011;27:155-64.

4. Odoom JK, Bel-Nono S, Rodgers D, Agbenohevi PG, Dafeamekpor CK, Sowa RML, et al. Troop education and avian 
influenza surveillance in military barracks in Ghana, 2011. BMC Public Health. 2012;12:957. http://dx.doi.org/10.1186/14712458-12-957

5. Agbenohevi PG, Odoom JK, Bel-Nono S, Nyarko EO, Alhassan M, Rodgers $\mathrm{D}$, et al. Biosecurity measures to reduce influenza infections in military barracks in Ghana. BMC Res Notes. 2015;8:14. http://dx.doi.org/10.1186/s13104-014-0956-0

6. Monne I, Meseko C, Joannis T, Shittu I, Ahmed M, Tassoni L, et al. Highly pathogenic avian influenza A (H5N1) virus in poultry, Nigeria, 2015. Emerg Infect Dis. 2015;21:1275-7. http://dx.doi.org/10.3201/eid2107.150421

7. World Organisation for Animal Health. Update on highly pathogenic avian influenza in animals (type $\mathrm{H} 5$ and $\mathrm{H} 7$ ). Last updated Aug 8 [cited 2016 Feb 5]. http://www.oie.int/animalhealth-in-the-world/update-on-avian-influenza/2016/

8. Horimoto T, Kawaoka Y. Reverse genetics provides direct evidence for a correlation of hemagglutinin cleavability and virulence of an avian influenza A virus. J Virol. 1994;68:3120-8.

9. Subbarao EK, London W, Murphy BR. A single amino acid in the PB2 gene of influenza A virus is a determinant of host range. J Virol. 1993;67:1761-4.

10. Gabriel G, Dauber B, Wolff T, Planz O, Klenk H-D, Stech J. The viral polymerase mediates adaptation of an avian influenza virus to a mammalian host. Proc Natl Acad Sci U S A. 2005;102:18590-5. http://dx.doi.org/10.1073/pnas.0507415102

Address for correspondence: Gülsah Gabriel, Heinrich-Pette-Institute, Leibniz Institute for Experimental Virology, Martinistr 52, Hamburg 20251 Germany; email: guelsah.gabriel@hpi.uni-hamburg.de

\section{Hepatitis E Virus in Yellow Cattle, Shandong, Eastern China}

\section{Bingyu Yan, ${ }^{1}$ Li Zhang, ${ }^{1}$ Lianfeng Gong, Jingjing Lv, Yi Feng, Jiaye Liu, Lizhi Song, Qing Xu, Mei Jiang, ${ }^{1}$ Aiqiang $X u$}

Author affiliations: Shandong University, Jinan, China (B. Yan, L. Zhang, J. Lv, Y. Feng, J. Liu, L. Song, Q. Xu, A. Xu); Shandong Center for Disease Control and Prevention, Jinan (B. Yan, L. Zhang, J. Lv, Y. Feng, J. Liu, L. Song, Q. Xu, A. Xu); Yantai Center for Disease Control and Prevention, Yantai, China (L. Gong, M. Jiang)

DOI: http://dx.doi.org/10.3201/eid2212.160641

To the Editor: Hepatitis E, caused by hepatitis E virus (HEV), is recognized as a zoonosis (1). HEV has been identified in a wide range of animals, and swine is the primary reservoir (2). In cattle, HEV strains have been recently described in yak (3), Holstein cows and their milk (4), and dairy cows in Xinjiang Province, China (5), but not in other cattle. Yellow cattle (Bos taurus), the predominant breed $(\approx 80 \%)$ in China ( 6 ), widely distributed over the country, and commonly used for meat and milk production or as a draft animal, could act as a potential HEV reservoir. The objective of this study was to determine whether HEV strains are circulating among yellow cattle in Shandong Province of eastern China.

During April-November 2011, a total of 842 blood samples from yellow cattle of local breeds were collected monthly as part of a severe fever with thrombocytopenia syndrome virus study. These samples were obtained from Laizhou and Penglai Counties ( $\approx 100 \mathrm{~km}$ apart) of Yantai Prefecture in Shandong Province.

Because the prevalent seasons for human HEV in this region were winter and spring, 254 samples (Laizhou $=131$; Penglai $=123$ ) collected only in April and November were selected for detection of HEV. All 254 cattle appeared to be healthy. Sixteen were $<1$ year of age, 108 were $1-3$ years of age, and 130 were $>3$ years of age. The cattle came from 20 villages (10 villages per county) and were raised by the local peasants, who owned an average of 2 cattle (range $1-8$ ). The animals were bred mainly to produce meat and seldom to produce milk.

Additional serum samples from domestic sheep, dogs, and chickens were also collected in this region simultaneously (online Technical Appendix Table 1, http://wwwnc. cdc.gov/EID/article/22/12/16-0641-Techapp1.pdf). All blood samples were centrifuged, and the separated serum was stored at $-70^{\circ} \mathrm{C}$ until use. The protocol for animal sampling was approved by the Animal Care Committee of the Chinese Center for Disease Control and Prevention.

We tested serum samples for total antibodies against HEV by using a double-antigen sandwich ELISA kit (Wantai Biological, Beijing, China) that uses a recombinant peptide of HEV open reading frame 2 (aa 394-606) from the virus as the antigen (7). Overall, the proportion seropositive for antibodies against HEV in yellow cattle was $47 \%(120 / 254 ; 95 \%$ CI $41 \%-54 \%)$, in line with the $28.2 \%$ positivity ratio previously reported in cattle from 26 provinces of China (8), suggesting that a high proportion of yellow cattle were exposed to HEV in this region. The proportions seropositive among sheep, dogs, and chickens were $32 \%(70 / 222), 41 \%(80 / 194)$, and $8 \%(41 / 484)$, respectively (online Technical Appendix Table 1).

We used nested reverse transcription PCR to amplify $644 \mathrm{nt}$ within HEV open reading frame 2 region, as described previously (9). We detected HEV RNA in 8 of 254 cattle samples; the overall proportion seropositive was $3 \%$. Positive yellow cattle included one $<1$ year of age, three $1-3$ years of age, and four $>3$ years of age. The 8 sequences obtained in this study (GenBank accession nos. KU904271, KU904273, KU904274, KU904278-KU904282) were subjected to phylogenetic analysis along with reference sequences for subtyping (10).

${ }^{1}$ These authors contributed equally to this article. 